\title{
Concurrent Anti-glomerular Basement Membrane Nephritis and IgA Nephropathy
}

\author{
Kwang-Sun Suh, Song-Yi Choi, Go Eun Bae, Dae Eun Choi ${ }^{1}$, Min-Kyung Yeo \\ Departments of Pathology and ${ }^{1}$ Nephrology, Chungnam National University School of Medicine, Daejeon, Korea
}

\begin{abstract}
Anti-glomerular basement membrane (GBM) nephritis is characterized by circulating anti-GBM antibodies and crescentic glomerulonephritis (GN) with deposition of IgG along the GBM. In a limited number of cases, glomerular immune complexes have been identified in anti-GBM nephritis. A 38-year-old female presented azotemia, hematuria, and proteinuria without any pulmonary symptoms. A renal biopsy showed crescentic GN with linear IgG deposition along the GBM and mesangial IgA deposition. The patient was diagnosed as concurrent anti-GBM nephritis and IgA nephropathy. Therapies with pulse methylprednisolone and cyclophosphamide administration were effective. Concurrent cases of both anti-GBM nephritis and IgA nephropathy are rare among cases of anti-GBM diseases with deposition of immune complexes. This rare case of concurrent anti-GBM nephritis and IgA nephropathy with literature review is noteworthy.
\end{abstract}

Key Words: Anti-glomerular basement membrane disease; Immunoglobulin A; Crescentic glomerulonephritis

Received: June 11, 2019 Revised: July 31, 2019 Accepted: August 5, 2019

Corresponding Author: Min-Kyung Yeo, MD, PhD, Department of Pathology, Chungnam National University School of Medicine, 266 Munhwa-ro, Jung-gu, Daejeon 35015, Korea Tel: +82-42-280-7196, Fax: +82-42-280-8199, E-mail: mkye083@gmail.com

Anti-glomerular basement membrane (GBM) disease manifests as rapidly progressive glomerulonephritis (RPGN) characterized by necrotizing glomerulonephritis (GN) with crescentic proliferation. The non-collagenous domain of the $\alpha-3$ chain of type IV collagen is identified as the autoantigen, and linear IgG deposition along the GBM aid diagnosis of anti-GBM disease. In rare cases, an association of anti-GBM disease with deposition of immune complexes has been reported. Herein, a case of concurrent anti-GBM nephritis and IgA nephropathy is presented.

\section{CASE REPORT}

A 38-year-old Korean woman noticed gross hematuria in April 2016. Laboratory analysis revealed a serum creatinine $(\mathrm{Cr})$ level of $0.65 \mathrm{mg} / \mathrm{dL}$; a serum total protein of $7.9 \mathrm{~g} / \mathrm{dL}$; a serum albumin of $4.4 \mathrm{~g} / \mathrm{dL}$; a serum anti-GBM antibody of $49 \mathrm{U} / \mathrm{mL}$; a urine spot protein/ $\mathrm{Cr}$ ratio of $0.360 \mathrm{~g} / \mathrm{g}$; and many urine spot erythrocytes. She was recommended further workup and treatment, but she refused. She experienced anorexia and weakness in June 2016. Her serum Cr level was $1.7 \mathrm{mg} / \mathrm{dL}$ at the second hospital visit. She was asked for hospitalization, but she refused treatment. She was admitted due to weakness and fever $\left(38.1^{\circ} \mathrm{C}\right)$ at the third hospital visit in July 2016. Laboratory analysis at this visit revealed a serum $\mathrm{Cr}$ level of $5.45 \mathrm{mg} / \mathrm{dL}$; a serum total protein of $7.5 \mathrm{~g} / \mathrm{dL}$; a serum albumin of $3.5 \mathrm{~g} / \mathrm{dL}$; a serum antiGBM antibody of $187.2 \mathrm{U} / \mathrm{mL}$; a urine spot protein/Cre ratio of $1.4 \mathrm{~g} / \mathrm{g}$; and many urine spot erythrocytes. Serum antinuclear antibody, anti-double stranded DNA, anti-neutrophil cytoplasmic antibody (ANCA), and antibodies to HIV, hepatitis B, and hepatitis $C$ were all negative. There was no evidence of lung involvement based on chest computed tomography and no respiratory symptoms.

A renal biopsy contained 16 glomeruli with five global scleroses, six fibrocellular crescents, and five cellular crescents (Fig. 1A). An immunofluorescence study showed linear deposition of IgG along the GBM (Fig. 1B) and granular deposition of IgA in mesangial spaces (Fig. 1C). Electron microscopy showed a diffusely wrinkled GBM and mesangial electron-dense deposition (Fig. 1D).

The pathologic diagnosis was "concurrent anti-GBM crescentic 
GN and IgA nephropathy," which was treated with intravenous methylprednisolone (500 mg/day for three successive days) with cyclophosphamide $(500 \mathrm{mg} /$ day) followed by oral prednisolone (50 mg/day). Plasmapheresis was avoided due to the possibility of side effects. Three months after the treatment, the anti-GBM antibody titer gradually decreased (anti-GBM titer, 15.6 U/mL) and renal function improved (Cr, $2.08 \mathrm{mg} / \mathrm{dL}$ ) (Fig. 2).

This study was approved by the Institutional Review Board of Chungnam National University Hospital with a waiver of informed consent (IRB No. 2016-11-009) and performed in accor-
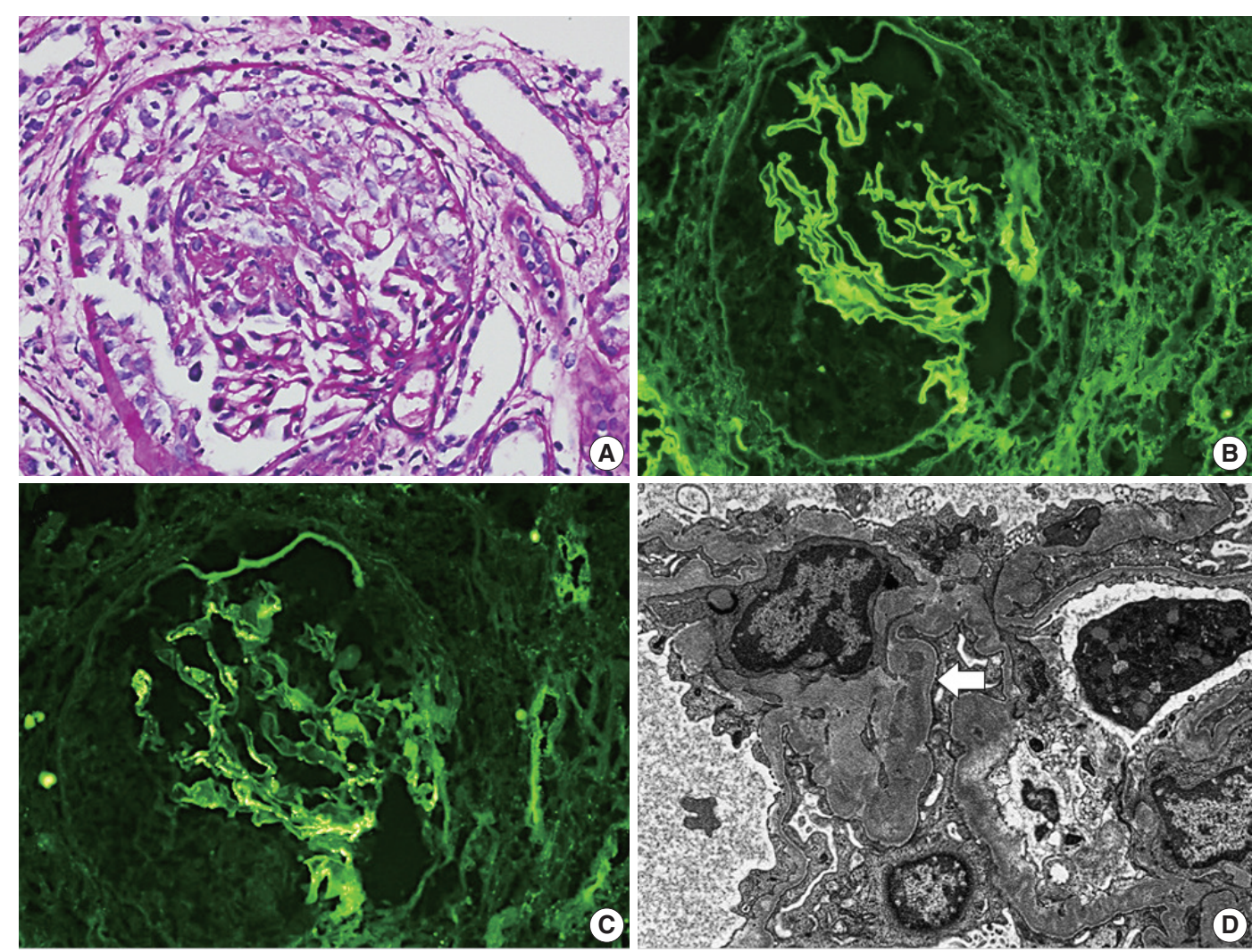

Fig. 1. (A) Light microscopy shows encircling cellular crescents (periodic acid-Schiff staining). Imunofluorescence shows linear deposition of IgG along the glomerular basement membrane (GBM) (B) and granular deposition of IgA in mesangial spaces (C). (D) Electron microscopy shows a diffusely wrinkled GBM and mesangial electron-dense deposition (arrow) (uranyl acetate/lead citrate staining, ×8,000).

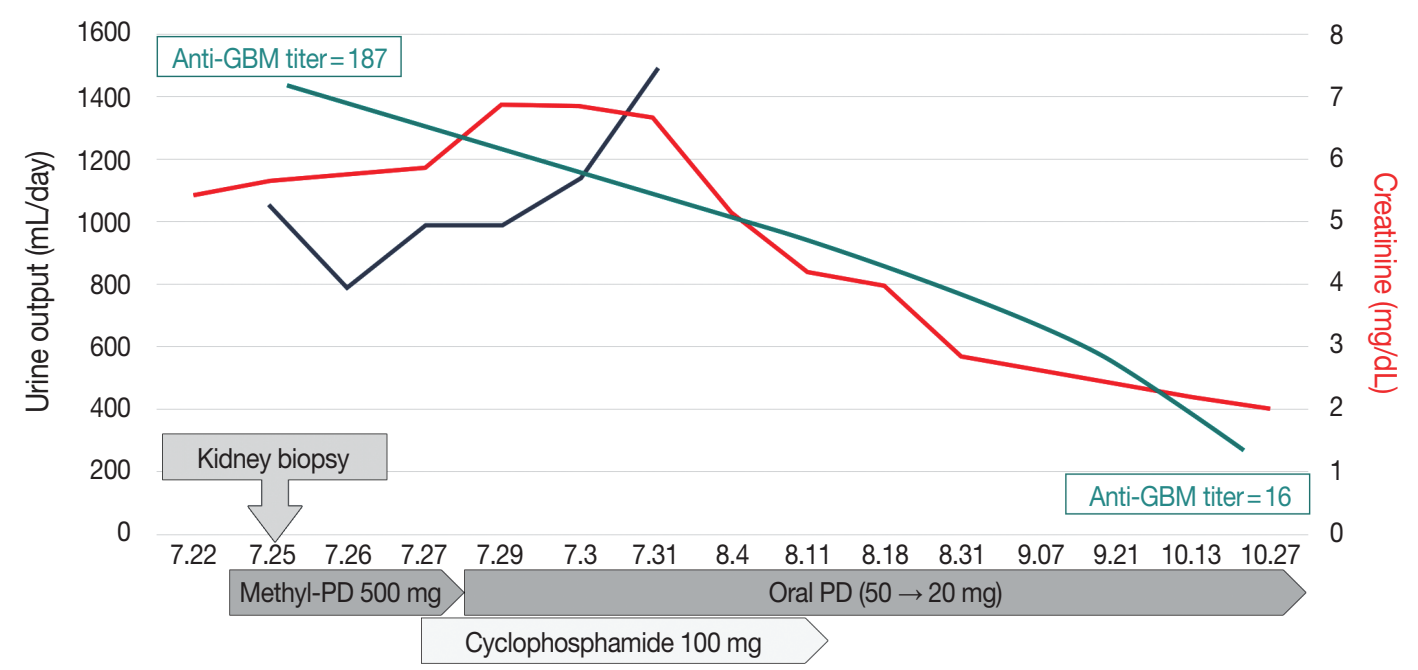

Fig. 2. Clinical course of the patient. GBM, glomerular basement membrane; PD, prednisolone. 
dance with the 1964 Helsinki declaration and its later amendments.

\section{DISCUSSION}

RPGN is classified into three categories as anti-GBM disease, immune complex disease, and ANCA-associated disease. Among these three categories, anti-GBM disease is the rarest and severest form of crescentic GN and is characterized by linear IgG deposition along the GBM with circulating anti-GBM antibodies. ${ }^{1}$ Overlapping features of crescentic GN are identified as coexistence of anti-GBM disease and ANCA antibodies, or anti-GBM disease and immune complex-mediated GN. ${ }^{2,3}$ ANCA antibodies are detected in $21 \%$ to $43 \%$ of anti-GBM disease patients and clinical characteristics of dual antibody-positive patients are documented. ${ }^{3,4}$ However, concurrent anti-GBM disease and immune complex-mediated GN is rarely reported and the most frequent form of combined immune complex $\mathrm{GN}$ is membranous

Table 1. Clinical characteristics of 10 cases (including the present case) of concurrent anti-GBM nephritis and lgA nephropathy in the literature

\begin{tabular}{lc}
\hline Characteristic & Percent \\
\hline Male:Female ratio & $4: 6(1: 1.5)$ \\
Age, mean (range, yr) & $43(27-60)$ \\
History of upper respiratory tract infection & 20 \\
History of hematuria or proteinuria & 40 \\
Symptoms or signs & \\
Hemoptysis or abnormal chest findings & 0 \\
Oliguria & 10 \\
Nephrotic syndrome & 20 \\
Laboratory findings & \\
Proteinuria, mean (g/day) & 2.6 \\
Hematuria & 100 \\
Creatinine, mean (mg/dL) & 5.0 \\
Anti-GBM antibody titer, mean (EU/mL) & 180 \\
Percentage of crescents, mean & 59 \\
Treatment & \\
Intravenous methyl-PD+Oral-PD & 100 \\
Cyclophosphamide & 60 \\
Mycophenolate mofetil & 20 \\
Methotrexate & 10 \\
Plasmapheresis & 30 \\
Prognosis & \\
Improved & \\
Not improved & 60 \\
Expired (due to gastrointestinal bleeding) & 40 \\
Dialysis & \\
Independent & \\
Dependent & \\
\hline & \\
\hline
\end{tabular}

GBM, glomerular basement membrane; PD, prednisolone. nephropathy. ${ }^{2}$ Concurrent anti-GBM disease and IgA nephropathy is rare and clinical characteristics are not well understood.

Herein, a case of concurrent anti-GBM nephritis and IgA nephropathy is presented. A literature review for concurrent antiGBM disease and IgA nephropathy using PubMed (http:// www.ncbi.nlm.nih.gov/pubmed) was performed resulting in identification of a total of nine cases from nine articles between 1995 and 2016 (Table 1, Supplementary Table S1)., ${ }^{2,-12}$

The average age and sex of concurrent anti-GBM disease and IgA nephropathy in reported cases was 43 years old with a male: female ratio of $1: 1.5$. Cases were reported in China $(n=5)$, the United States $(n=2)$, Canada $(n=1)$, and Japan $(n=1)$. None of the cases was previously diagnosed as immune complex GN including $\operatorname{Ig}$ A nephropathy. A recent history of hematuria or proteinuria was shown in $40 \%$ of cases and a history of upper respiratory tract infection was present in $20 \%$ of cases. None showed symptoms of hemoptysis or pulmonary involvement based on image analysis. Oliguria was seen in $10 \%$ of cases. The mean proteinuria, serum $\mathrm{Cr}$ levels, and anti-GBM antibody titer (among available cases) were $2.6 \mathrm{~g} / \mathrm{day}, 5.0 \mathrm{mg} / \mathrm{dL}$, and $180 \mathrm{EU} /$ $\mathrm{mL}$. The ANCA autoantibody was not identified. The percentage of fibrocellular or cellular crescents in renal biopsies was $59 \%$. All cases were treated with steroids and some cases with combined cytotoxic immunosupressive agents, including cyclophosphamide, mycophenolate mofetil, or methotrexate. Plasmapheresis was applied to $30 \%$ of cases. More than half (60\%) of cases showed improved renal function and dialysis dependence was shown in $30 \%$ of cases.

Cui et al. ${ }^{2}$ compared clinical and laboratory data of patients suffering anti-GBM disease associated with depositions of immune complexes (10 cases) with data from anti-GBM disease alone (37 cases). No significant differences in clinical and pathological findings were observed. In comparison with anti-GBM disease associated with depositions of immune complexes reported by Cui et al., ${ }^{2}$ this present review of cases with concurrent antiGBM disease and IgA nephropathy showed a tendency of better prognosis. Symptoms of oliguria were reported less commonly in concurrent anti-GBM disease and IgA nephropathy (10\%) than in anti-GBM disease with depositions of immune complexes (40\%). Percentage of crescent formation in cases of concurrent anti-GBM disease and IgA nephropathy (59\%) is less than that in cases of anti-GBM disease with deposition of immune complexes $(93.8 \%)$. Dialysis independence was more frequent in concurrent anti-GBM disease and IgA nephropathy (60\%) than in anti-GBM disease with immune complexes (10\%).

The connection between anti-GBM disease and IgA nephrop- 
athy is uncertain. $\operatorname{IgA}$ nephropathy is the most common immune complex-mediated GN and clinical features vary from asymptomatic hematuria to RPGN. ${ }^{13}$ Occurrence of anti-GBM disease superimposed on underlying symptomatic IgA nephropathy could be a possible explanation. No cases were confirmed as IgA nephropathy before diagnosis of anti-GBM nephritis in the present review; however, a history of upper respiratory tract infection or microscopic hematuria was noted in some cases. Pathological changes of GBM components triggered by IgA-related immune complex deposition is another hypothesis. ${ }^{14}$ Anti-GBM antibodies might alter the permeability of GBM to allow circulating immune complex deposition in the mesangium. ${ }^{2}$

Based on the present review of a limited number of cases, concurrent anti-GBM disease and IgA nephropathy seems to have a better prognosis than anti-GBM disease alone or anti-GBM disease with immune complexes. In order to understand concurrent anti-GBM disease and IgA nephropathy as more than just a simple coincidence of the two diseases, more cases need to be analyzed with an in-depth examination of underlying pathogenic relationships between anti-GBM disease and IgA nephropathy.

\section{Electronic Supplementary Material}

Supplementary materials are available at Journal of Pathology and Translational Medicine (https://jpatholtm.org).

\section{ORCID}

Kwang-Sun Suh: https://orcid.org/0000-0003-3874-864X

Song-Yi Choi: https://orcid.org/0000-0002-8496-5613

Go Eun Bae: https://orcid.org/0000-0001-9288-1383

Dae Eun Choi: https://orcid.org/0000-0003-2870-3958

Min-kyung Yeo: https://orcid.org/0000-0001-8873-0021

\section{Author Contributions}

Conceptualization: KSS, MKY.

Resources: SYC, DEC.

Supervision: GEB.

Writing_original draft: MKY.

Writing_review and editing: MKY, KSS.

\section{Conflicts of Interest}

The authors declare that they have no potential conflicts of interest.

\section{Funding}

This work was supported by Chungnam National University Hospital Research Fund, 2018.

\section{REFERENCES}

1. Jennette JC, D'Agati VD, Olson JL, Silva FG. Heptinstall's pathology of the kidney. Philadelphia: Lippincott Williams \& Wilkins, 2014; 661-2.

2. Cui Z, Zhao MH, Wang SX, Liu G, Zou WZ, Wang HY. Concurrent antiglomerular basement membrane disease and immune complex glomerulonephritis. Ren Fail 2006; 28: 7-14.

3. Rutgers A, Slot M, van Paassen P, van Breda Vriesman P, Heeringa $\mathrm{P}$, Tervaert JW. Coexistence of anti-glomerular basement membrane antibodies and myeloperoxidase-ANCAs in crescentic glomerulonephritis. Am J Kidney Dis 2005; 46: 253-62.

4. Alchi B, Griffiths M, Sivalingam M, Jayne D, Farrington K. Predictors of renal and patient outcomes in anti-GBM disease: clinicopathologic analysis of a two-centre cohort. Nephrol Dial Transplant 2015; 30: 814-21.

5. Trpkov K, Abdulkareem F, Jim K, Solez K. Recurrence of anti-GBM antibody disease twelve years after transplantation associated with de novo IgA nephropathy. Clin Nephrol 1998; 49: 124-8.

6. Wechsler E, Yang T, Jordan SC, Vo A, Nast CC. Anti-glomerular basement membrane disease in an HIV-infected patient. Nat Clin Pract Nephrol 2008; 4: 167-71.

7. Wang A, Wang Y, Wang G, Zhou Z, Xun Z, Tan X. Mesangial IgA deposits indicate pathogenesis of anti-glomerular basement membrane disease. Mol Med Rep 2012; 5: 1212-4.

8. Yamaguchi H, Takizawa H, Ogawa Y, Takada T, Yamaji I, Ura N. A case report of the anti-glomerular basement membrane glomerulonephritis with mesangial IgA deposition. CEN Case Rep 2013; 2: 6-10.

9. Gao B, Li M, Xia W, Wen Y, Qu Z. Rapidly progressive glomerulonephritis due to anti-glomerular basement membrane disease accompanied by IgA nephropathy: a case report. Clin Nephrol 2014; 81: $138-41$.

10. Troxell ML, Houghton DC. Atypical anti-glomerular basement membrane disease. Clin Kidney J 2016; 9: 211-21.

11. Ge YT, Liao JL, Liang W, Xiong ZY. Anti-glomerular basement membrane disease combined with IgA nephropathy complicated with reversible posterior leukoencephalopathy syndrome: an unusual case. Am J Case Rep 2015; 16: 849-53.

12. $\mathrm{Xu} \mathrm{D}, \mathrm{Wu}$ J, Wu J, et al. Novel therapy for anti-glomerular basement membrane disease with IgA nephropathy: a case report. Exp Ther Med 2016; 11: 1889-92.

13. Emancipator SN, Lamm ME. IgA nephropathy: pathogenesis of the most common form of glomerulonephritis. In: Rubin E, Damjanov I, eds. Pathol reviews. Clifton: The Humana Press, 1990; 113-28.

14. Kamimura H, Honda K, Nitta K, et al. Glomerular expression of alpha2(IV) and alpha5(IV) chains of type IV collagen in patients with IgA nephropathy. Nephron 2002; 91: 43-50. 